\title{
Gedanken zu \\ „fachbezogenen Referenzsystemen“
}

\author{
Hans-Christoph Grunau
}

\begin{abstract}
Aus den aktuellen Bemühungen der ASIIN um strategische Partnerschaften mit Fakultätentagen könnten meines Erachtens fehlgeleitete „fachbezogene Referenzsysteme“ hergeleitet werden, die uns am Ende das tägliche Akkreditierungsleben schwer und Experimente oder einfach nur Nicht-Standard-Studiengänge unmöglich machen. „ASIIN-FEH für alle“, ich hätte nicht gedacht, dass das nach dem BVG-Urteil von 2016 jemals noch möglich werden könnte. Die aktuelle Entwicklung in MNFT und KMathF halte ich für besorgniserregend in Sachen akademischer Freiheit.
\end{abstract}

Was sind und was sollen "fachbezogene Referenzsysteme?"

Seit gut zwei Jahren wird unter anderem auf den Plenarversammlungen der Konferenz der mathematischen Fachbereiche (KMathF) über „fachbezogene Referenzsysteme“ diskutiert. Viele der Leserinnen und Leser mögen sich wundern, durch welche veränderten Rahmenbedingungen diese Diskussion initiiert wurde. Immerhin befassen wir uns alle seit nunmehr gut 20 Jahren mit dem BolognaProzess; und bis 2016 hatte ich den Eindruck, dass alle Grundsatzdiskussionen geführt worden seien.

In der Tat hat sich aber seit dem Beschluss des Bundesverfassungsgerichts vom 17. Februar 2016 der rechtliche Rahmen verändert. Die Bewertung von Inhaltlichkeit ist nun im Rahmen von Akkreditierungsverfahren nicht nur zulässig, sondern ausdrücklich erforderlich, und hierbei sollen Begutachtende so genannte „fachbezogene Referenzsysteme" beachten. Diesbezüglich ist ein Diskurs-Vakuum entstanden, das verschiedene Akteure, u.a. Fachgesellschaften, Fakultätentage und die Akkreditierungsagentur ASIIN, zu füllen versuchen.

Diese „fachbezogenen Referenzsysteme“ werden vermutlich normative Wirkung entfalten - auch an systemakkreditierten Hochschulen. Die Diskussionen auf den letzten Plenarversammlungen der KMathF habe ich so wahrgenommen, dass hier möglicherweise Positionspapiere verabschiedet werden könnten, die stark regulatorisch wirken und Handlungsspielräume einschränken könnten, die unser Fach in den letzten Jahren erkannt und genutzt hat. Die Vorlage zur letzten Plenarversammlung ${ }^{1}$ basiert ganz offen auf den fachspezifischen ergänzenden Hinweisen (FEH) der ASIIN. Diese könnten also künftig sogar Wirkung in allen Akkreditierungsverfahren in der Mathematik entfalten, unabhängig von der Wahl der Akkreditierungsagentur und selbst an systemakkreditierten Hochschulen.

Das Ziel dieses Beitrags besteht darin, eine möglichst breite und vielfältige Diskussion anzustoßen, damit wir am Ende Positionsbeschreibungen haben, in denen sich nach Möglichkeit alle wiederfinden können. Im zweiten Teil dieses Beitrags möchte ich für einen Ansatz für „fachbezogene Referenzsysteme“ werben, der Handlungsspielräume erhält und erweitert und gleichzeitig relativ genau beschreibt, welche fachlichen Fähigkeiten Studierende der Mathematik erwerben (sollen). Diese Gedanken beruhen auf Diskussionen, die wir in der Magdeburger Mathematik geführt und in einem Fakultätsratbeschluss niedergelegt haben. Ob diese in ein mögliches Positionspapier eingehen werden, wird sich zeigen.

Im ersten Teil stelle ich aber einige Fakten zusammen, inwieweit sich der rechtliche Rahmen in den letzten drei Jahren verändert hat.

\section{Der veränderte rechtliche Rahmen}

In der Pressemitteilung des Bundesverfassungsgerichts zu seinem Beschluss vom 17 . Februar $2016^{2}$ heißt es $u$. a.:

Das Grundrecht der Wissenschaftsfreiheit steht zwar Vorgaben zur Qualitätssicherung von Studienangeboten grundsätzlich nicht entgegen. Wesentliche Entscheidungen zur Akkreditierung von Studiengängen darf der Gesetzgeber jedoch nicht anderen Akteuren überlassen. [...] Die Agenturen machen zudem Vorgaben zur prozentualen Zusammensetzung der Inhalte von Lehrplänen, zu den Studien- und Prüfungsordnungen und sprechen Empfehlungen zur Benennung von Studienschwerpunkten und Modulen aus. Damit erfasst die Akkreditierung unmittelbar Form und Inhalt wissenschaftlicher Lehre. [...] Dieser Eingriff in die Wissenschaftsfreiheit lässt sich verfassungsrechtlich nicht rechtfertigen.

Die hier festgestellten Mängel wurden schnell behoben durch:

- Staatsvertrag 3 über die Organisation eines gemeinsamen Akkreditierungssystems zur Qualitätssicherung in Studium und Lehre an deutschen Hochschulen, Juni 2017. 
Durch nachfolgend verabschiedete Ländergesetze hat dieser Staatsvertrag zum 1. Januar 2018 Gültigkeit erlangt.

- Musterrechtsverordnungen ${ }^{4}$ : Beschluss der Kultusministerkonferenz vom 7.12.2017.

Diese wurden nachfolgend in Rechtsverordnungen der Länder umgesetzt. Sie ersetzen, präzisieren und ergänzen die bisherigen ländergemeinsamen Strukturvorgaben der KMK.

Dadurch ergeben sich einige Änderungen im Akkreditierungsalltag etwa dahingehend, dass Akkreditierungsagenturen in solchen Verfahren nur noch als Dienstleister auftreten, die Begehungen und Begutachtungen organisieren und dokumentieren. Die Vorgänge werden dann dem Akkreditierungsrat vorgelegt, wo die Akkreditierungsentscheidung getroffen wird. Dieses betrifft Programm- und Systemakkreditierungen gleichermaßen und wird den Alltag der Akkreditierungspraxis vermutlich nur am Rande verändern.

Deutlich stärkere Auswirkungen aber wird haben, dass der Staatsvertrag und die nachgeordneten Rechtsverordnungen der Länder die Inhaltlichkeit von Akkreditierungsverfahren stärken. Hierzu heißt es in Artikel 2, Absatz 3 des Staatsvertrags:

$\mathrm{Zu}$ den fachlich-inhaltlichen Kriterien gehören [... ] auf dem aktuellen Stand von Wissenschaft und Forschung befindliche fachlich-inhaltliche Standards, $[\ldots]$.

Für Details verweist der Staatsvertrag auf Rechtsverordnungen der Länder. Hierzu heißt es in §13 „FachlichInhaltliche Gestaltung der Studiengänge“ der Musterrechtsverordnung in Absatz 1:

Die Aktualität und Adäquanz der fachlichen und wissenschaftlichen Anforderungen ist gewährleistet. Die fachlich-inhaltliche Gestaltung und die methodischdidaktischen Ansätze des Curriculums werden kontinuierlich überprüft und an fachliche und didaktische Weiterentwicklungen angepasst. Dazu erfolgt eine systematische Berücksichtigung des fachlichen Diskurses auf nationaler und gegebenenfalls internationaler Ebene.

Warum der Gesetzgeber diese Regelung so weit fasst, erläutert er im Begründungstext, der der oben genannten Quelle anhängt:

Mit Blick auf Artikel 5 Absatz 3 Grundgesetz beschränkt sich die Regelung auf die Prüfung der Einhaltung prozessualler Erfordernisse zur Sicherstellung eines fachlich fundierten Studiengangkonzepts und lässt den Gutachterinnen und Gutachtern inhaltlich einen weiten Ermessensspielraum. [...] Dazu gehören die kritische Reflexion unterschiedlicher fachbezogener Referenzsysteme ebenso wie die kontinuierliche Auseinandersetzung mit dem neuesten Stand der Forschung.
Dieses definiert die fachbezogenen Referenzsysteme: Dabei handelt es sich u. a. um die zusammengefasste Darstellung eines solchen fachlichen Diskurses. Im Falle der Mathematik kann dieses beispielsweise durch KMathF, MNFT, DMV, GAMM und bei Lehramtsbezug auch durch GDM und MNU erfolgen.

\section{Ausgestaltung der fachbezogenen Referenzsysteme}

Hierzu gehen die Meinungen aktuell weit auseinander. Auf der einen Seite gibt es die Diskussionsvorlage (Fundstelle s. o.) einer kleinen Arbeitsgruppe der KMathF und eines Vertreters des DMV-Präsidiums, die die FEH der ASIIN zu einem solchen fachbezogenen Referenzsystem weiterentwickeln möchte. Ich empfinde dieses Papier als regulatorisch, konservativ bis rückwärtsgewandt und hinsichtlich der Festlegung gewisser Leistungspunktanzahlen für bestimmte curriculare Elemente als nicht konform mit Inhalt und Geist des Beschlusses des Bundesverfassungsgerichts. Auf jeden Fall würde ein solches Papier neue Mathematikstudiengänge, in denen ein zweites MINT-Fach auf Augenhöhe mit Mathematik kombiniert werden könnte, verhindern bzw. als Nicht-Mathematik ausgrenzen. Auch die Durchlässigkeit zwischen verschiedenen MINT- und Lehramtsstudiengängen etwa beim Übergang von Bachelor zu Master würde verhindert. Meines Erachtens würden wir mit dem ersten Punkt viele junge Menschen vor den Kopf stoßen, die „irgendetwas mit Mathe, Physik, Informatik oder Technik“ studieren wollen. Mit dem zweiten Punkt würden wir einen der großen Vorteile des Bachelor-Master-Systems dezidiert nicht nutzen wollen. Die Diskussion dieser Vorlage am 29.06. auf der KMathF-Plenarversammlung in Bochum war kontrovers, aber meines Erachtens viel zu kurz, um auch nur den wichtigsten Facetten der vielschichtigen Thematik gerecht werden zu können. Es gab ein - bei weitem nicht konsensuales - Signal an die Arbeitsgruppe, den eingeschlagenen Weg weiterzuverfolgen.

Auf der anderen Seite diskutieren wir in Magdeburg seit einigen Jahren einen anderen Ansatz, und je länger dieser Ansatz in andere Fachbereiche hinein diffundiert, umso mehr positive Rückmeldungen erhalten wir dazu. Die Idee besteht darin, Mathematikstudiengänge „vom Ende“ her zu konzipieren bzw. zu bewerten. Meines Erachtens sollte die Mathematik die aktuelle Dynamik nutzen und jetzt in eine inhaltliche Diskussion einsteigen:

An welchen Fähigkeiten kann ich eine/n Mathemati$\mathrm{ker} /$ in erkennen? Was muss ein/e Mathematiker/in können?

Im Folgenden werde ich statt „Fähigkeiten“ das synonyme Wort „Kompetenzen“ verwenden. Dieses ist auf Grund unangenehmer Erfahrungen zwar oft negativ besetzt. Aber in den letzten Jahren hat sich durchgesetzt, dass damit auch „Fachkompetenzen“ gemeint sind. Die Leserinnen und Leser, die sich mit diesem Terminus gar nicht anfreunden können, mögen diesen für sich durch „fachliche Fähigkeiten" ersetzen. 
Fachkompetenzorientierung versus Festlegung von Vorlesungskanons

Immer wieder wird als Argument für ein Mathematikstudium gebracht, „man lerne strukturiert und strukturierend zu arbeiten." Meines Erachtens ein klares Bekenntnis zur Kompetenzorientierung des Mathematikstudiums.

In keinem Vorstellungsgespräch wird man fragen: „Haben Sie die Vorlesungen X, Y, Z belegt?“ Sondern: „Können Sie mit diesem und jenem umgehen?"

Wichtig ist also, was die Absolventinnen und Absolventen können. Natürlich müssen sie dazu auch etwas wissen, aber der genaue Umfang ihres Fachwissens oder gar der belegten Veranstaltungen ist dabei eher nachrangig. Die Fähigkeit zu stukturiertem Denken alleine charakterisiert Mathematiker/innen aber nur unzureichend, denn dieses lernt man beispielsweise auch in einem Latein- oder Philosophiestudium. Es lohnt sich also die Mühe zu überlegen, was Absolvent/inn/en von Mathematikstudiengängen mit in ihr Berufsleben nehmen, das sie nicht ebenso gut in einem Informatik-, Physik-, Philosophie- oder Linguistikstudium hätten erwerben können.

Bevor ich nun zu der eigentlichen Frage „Was muss ein/eine Mathematiker/in können?" komme, ist es vielleicht zielführend, sich zu überlegen, welche klassischen und modernen Konzepte die Mathematik wirklich vorangebracht haben.

Welche grundlegenden Konzepte prägen die Mathematik?

Ohne Anspruch auf Vollständigkeit werden hier einige grundlegende Konzepte der Mathematik zusammengestellt, die innerhalb und außerhalb der Mathematik besonders große Wirkungen entfaltet haben:

- Quantitative Behandlung von Geometrie durch Einführung von Koordinaten.

- Differential- und Integralrechnung, entwickelt als Werkzeug zur Modellierung physikalischer, naturwissenschaftlicher, technischer und geometrischer Sachverhalte.

○ Entwicklung einer präzisen und weitgehend formalisierten Fachsprache.

- Erkennen von Strukturen, zunächst und insbesondere algebraischer Strukturen. Axiomatisches Arbeiten.

- Isomorphismen in den verschiedensten Kontexten, Invarianten von Isomorphieklassen.

- Vollständigkeit von Zahlenräumen, insbesondere aber auch Funktionenräumen, deren Normen durch anwendungsorientierte (Energie-) Integralterme induziert werden.

- Kompaktheit.

○ Quantitative Modellierung von „Zufall“ und Analyse „zufälliger" Daten.

- Analyse von Problemen und Beweisen bzgl. grundsätzlicher Algorithmisierbarkeit und ggfs. strukturierte Entwicklung entsprechender Algorithmen.

- Nutzung leistungsfähiger Rechner zur effizienten (exakten) Lösung „großer" diskreter Probleme.
○ Nutzung leistungsfähiger Rechner zur effizienten approximativen Lösung "großer" kontinuierlicher Probleme.

$\circ \ldots$

$\circ \ldots$

$\circ \ldots$

Diese Liste soll eine Diskussion stimulieren, ob die genannten Konzepte alle als grundlegend angesehen werden und wodurch diese Liste noch ergänzt werden sollte.

Was muss ein/eine Mathematiker/in können?

Meines Erachtens erkennt man Mathematiker/innen daran, dass diese mit einem relevanten Anteil (etwa die Hälfte?) der vorher genannten grundlegenden Konzepte sicher umgehen können. Dieses schließt innermathematische Wechselwirkungen bzw. außermathematische Anwendungen ein. Auf Bachelorniveau sollte man sicheres Anwenden bestehender Konzepte erwarten, während auf Masterniveau neben einem höheren Abstraktions- oder Komplexitätsgrad auch weitergehend Entwickeln von Konzepten, Zusammenhängen und/oder Anwendungen erwartet werden kann.

Aus den Qualifikationszielen die inhaltliche Gestaltung von Studiengängen ableiten

Die Diskussion der letzten Jahre über learning outcomes und Kompetenzorientierung postuliert ein deduktives Vorgehen für die Gestaltung von Studiengängen: Aus den Qualifikationszielen sollen die Curricula abgeleitet werden. Mathematiker/inne/n sollte dieses auf Grund ihres axiomatischen Vorgehens grundsätzlich sehr entgegenkommen. Es lohnen sich Gedankenspiele, welche curricularen Erfordernisse sich aus den oben definierten Qualifikationszielen ergeben. Im Ergebnis wird man feststellen, dass es viele verschiedene Möglichkeiten gibt, Mathematikstudiengänge zielführend zu implementieren - was ein Blick über die Landesgrenzen z. B. in den angelsächsischen Raum auch bestätigt.

Ein großes Missverständnis entstünde nun, wenn man versuchte, die genannten Konzepte und Kompetenzen bestimmten Vorlesungen oder Modulen zuzuordnen. Exemplarisch sei hierzu das Konzept der Vollständigkeit genannt. Den Umgang hiermit kann man in AnalysisVorlesungen ( $C^{k}$ - und $L^{p}$-Räume), aber auch in der Funktionalanalysis, in einem Kurs über partielle Differentialgleichungen oder einer entsprechenden Numerikveranstaltung erlernen. Aus scheinbar ganz anderer Sicht kann diese Kompetenz aber auch im Bereich der algebraischen Zahlentheorie erworben werden.

Oft wird als Argument für curriculare Einheitlichkeit unter anderem die Gewährleistung von Mobilität genannt. Auch hier hilft meines Erachtens ein kompetenzorientierter Ansatz weiter. Welche Erkenntnis gewinnen wir daraus, dass Studierende etwa Analysis I-III belegt haben, so verschieden diese Vorlesungen aufgebaut sein mögen? Meines Erachtens ist da z. B. die Frage viel wichtiger, ob 
die Studierenden mit dem abstrakten Konzept, Realisierungen und Anwendungen vollständiger Funktionenräume umgehen können, oder ob sie eher differentialgeometrische bzw. global-analytische Konzepte beherrschen.

Anhand der Qualifikationsziele und deren curricularer Umsetzung Studiengänge inhaltlich bewerten

Der vorhergehende Absatz hat deutlich werden lassen, dass es sehr viele Möglichkeiten gibt, die genannten mathematischen Kernkompetenzen in ganz unterschiedlich konzipierten Curricula zu vermitteln.

Meines Erachtens sollten im Rahmen von Qualitätssicherungsverfahren mathematische Studiengänge da- nach bewertet werden, inwiefern die Studierenden die Fähigkeit erwerben, mit den oben genannten grundlegenden mathematischen Konzepten auf verschiedenen Abstraktions- und Komplexitätsniveaus sicher umzugehen.

Natürlich gibt es sehr gute Mathematikstudiengänge, die dem aktuellen Diskussionspapier der KMathF entsprechen. Dieser Weg ist aber meines Erachtens nicht der einzig gangbare - und diese sehr guten Mathematikstudiengänge würden auch nach dem vorgeschlagenen Bewertungsmodus als solche erkannt.

Meines Erachtens sollte Vielfalt mathematischer Studienangebote als Bereicherung empfunden werden. Mobilität wird durch den Erwerb gleichwertiger Kompetenzen ermöglicht. Eine europa- oder gar weltweite Vereinheit-

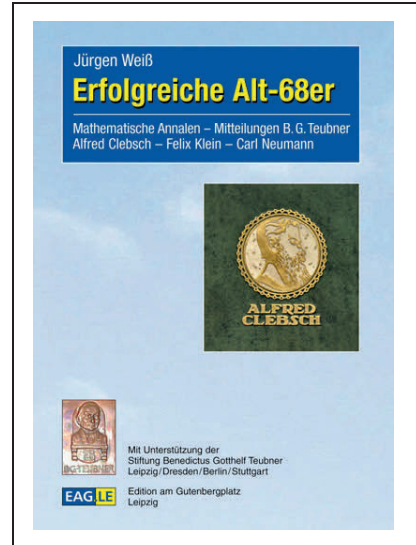

J. Weiß. EAGLE 101.

1. A. 2018. 978-3-95922-101-6

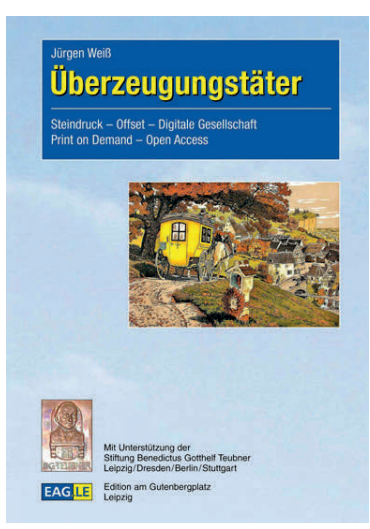

J. Weiß. EAGLE 100

1. A. 2017. 978-3-95922-100-9

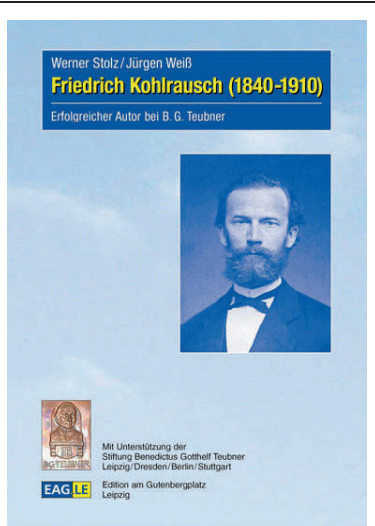

W. Stolz / J. Weiß. EAGLE 095. 1. A. 2017. 978-3-95922-095-8

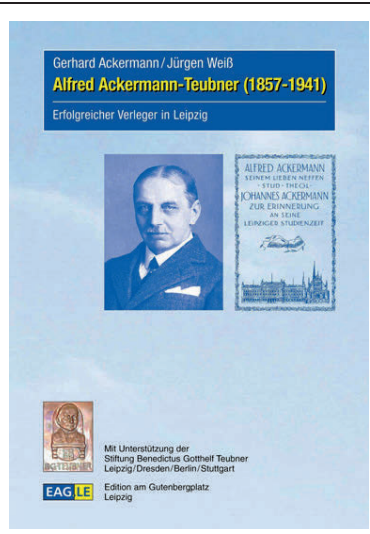

G. Ackermann / J. Weiß. EAGLE 090 . 1. A. 2016. 978-3-95922-090-3

\section{Leipzig, 21.02.1811: Firmengründung durch Benedictus Gotthelf Teubner (1784-1856). \\ Leipzig, 21.02.2003: Gründung der Teubner-Stiftung und des Verlages Edition am Gutenbergplatz Leipzig (EAGLE) / www.eagle-leipzig.de Leipzig, 21.02.2020: Vierter Wissenschaftspreis der Teubner-Stiftung zur Förderung der Mathematischen Wissenschaften.}

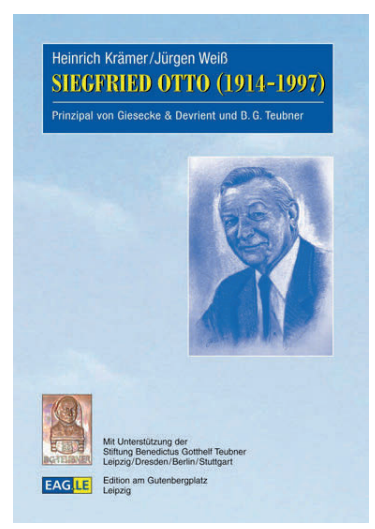

H. Krämer / J. Weiß. EAGLE 075. 1. A. 2014. 978-3-937219-75-2

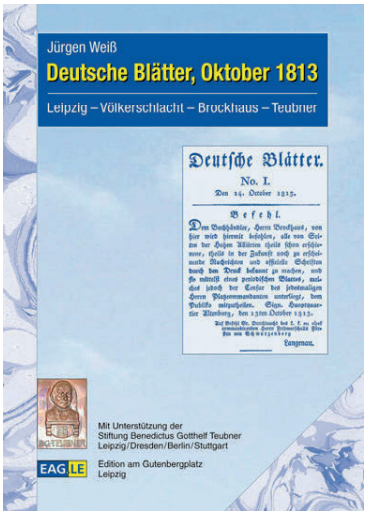

J. Weiß. EAGLE 065.

1. A. 2013. 978-3-937219-65-3

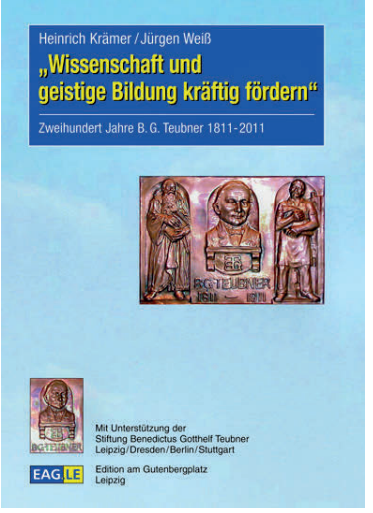

H. Krämer / J. Weiß. EAGLE 050. 1. A. 2011. 978-3-937219-50-9

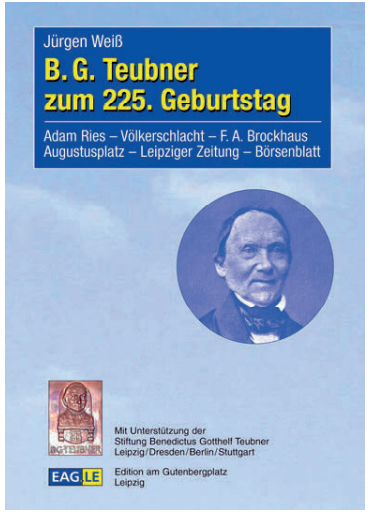

J. Weiß. EAGLE 035.

1. A. 2009. 978-3-937219-35-6 
lichung der Curricula - so wenig realistisch und wünschenswert diese ist - würde Mobilität auch nur scheinbar erleichtern, denn wozu wechseln, wenn überall dasselbe geboten wird?
Anmerkungen

1. tinyurl.com/y5bwbvr4

2. tinyurl.com/yyrec6r2

3. tinyurl.com/y5k2k27y

4. tinyurl.com/y2nkxvza

Prof. Dr. Hans-Christoph Grunau Otto-von-Guericke-Universität Magdeburg

Universitätsplatz 2, 39106 Magdeburg

hans-christoph.grunau@ovgu.de

\begin{abstract}
Hans-Christoph Grunau wurde 1961 in Wuppertal geboren. Er hat 1990 in Göttingen promoviert und sich 1996 in Bayreuth habilitiert. Nach einer weiteren Station in Utrecht wurde er 2001 Professor in Magdeburg. 2004-2008 war er Studiendekan, 2008-2016 Herausgeber des Jahresberichts der DMV und ist seit 2016 Dekan der Magdeburger Fakultät für Mathematik. Sein Arbeitsgebiet sind partielle Differentialgleichungen und Variationsrechnung mit Bezügen zur Differentialgeometrie.
\end{abstract}

\title{
Einladung zur Mitgliederversammlung der DMV
}

\section{Karlsruhe, 24.9.2019}

Im Auftrag des Präsidenten der Deutschen MathematikerVereinigung, Friedrich Götze, lade ich alle DMV-Mitglieder herzlich ein, an unserer Mitgliederversammlung während der Jahrestagung der DMV in Karlsruhe (23.26. 9. 2019) teilzunehmen.

Die Mitgliederversammlung der DMV findet am Dienstag, dem 24. 9. 2019, von 17-18 Uhr im Seminarraum 1.067, Englerstraße 2, Gebäude 20.30, Karlsruher Institut für Technologie, 76131 Karlsruhe, statt.
Tagesordnung

TOP 1: Genehmigung der Tagesordnung

TOP 2: Bericht des Präsidenten

TOP 3: Format der Jahrestagung

TOP 4: Minkowski-Medaille

TOP 5: Diskussion in der Mitgliederversammlung

TOP 6: Kassenbericht, Bericht der Kassenprüfer, Entlastung des Vorstands

TOP 7: Wahl der Kassenprüfer

TOP 8: Anstehende Jahrestagungen

TOP 9: Verschiedenes

Informationen zu TOP 4 finden Sie im Grußwort dieses Heftes auf S. 57. Ich freue mich darauf, Sie in Karlsruhe zu begrüßen. Daniel Grieser (Schriftführer der DMV) 